\title{
Electrochemical deposition and thermoelectric characterisation of a semiconducting 2-D metal-organic framework thin film.
}

Maria de Lourdes Gonzalez-Juarez ${ }^{a}$, Eduardo Flores ${ }^{b}$, Marisol Martin-Gonzalez ${ }^{b}$, Iris Nandhakumar*a, and Darren Bradshaw*a

aSchool of Chemistry, University of Southampton, Southampton SO17 1BJ, UK

b'Instituto de Micro y Nanotecnología (IMN-CNM-CSIC), C/ Isaac Newton 8, PTM, E-28760 Tres Cantos, Spain

Email: D.Bradshaw@soton.ac.uk; I.Nandhakumar@soton.ac.uk

\begin{abstract}
:
The electrical conductivity and porosity of the 2-dimensional metal-organic framework $\mathrm{Cu}_{3}(2,3,6,7,10,11 \text {-hexahydroxytriphenylene })_{2}\left[\mathrm{Cu}_{3}(\mathrm{HHTP})_{2}\right]$ make it a promising candidate for thermoelectric applications. In this work, we report the electrochemical synthesis of $\mathrm{Cu}_{3}(\mathrm{HHTP})_{2}$ films by an anodization approach and an evaluation of its thermoelectric properties. The electrochemically synthesised $\mathrm{Cu}_{3}(\mathrm{HHTP})_{2}$ thin films were transferred using a wet chemical method in order to perform electrical measurements. We are reporting the first thermoelectric measurements of this framework both in bulk and thin film form which resulted in Seebeck coefficients of $-7.24 \mu \mathrm{V} / \mathrm{K}$ and $-121.4 \mu \mathrm{V} / \mathrm{K}$ with a power factor of $3.15 \times 10^{-3} \mu \mathrm{W} \mathrm{m} \mathrm{m}^{-1}$ for the film respectively. The negative Seebeck coefficients suggest that $\mathrm{Cu}_{3}(\mathrm{HHTP})_{2}$ behaves as an n-type semiconductor. Since the introduction of conductive MOFs in electronic devices is at an early stage of development, we believe this work validates the use of electrochemical synthetic routes of conducting MOFs to further investigate and expand their applications in thin film technologies.
\end{abstract}




\section{Introduction:}

In recent years, there has been special interest in developing devices capable of harvesting and storing energy from natural resources without the generation of pollution. A recent manner to harvest energy, especially in those environments in which heat waste is involved, is through thermoelectric (TE) materials. It has been reported that in gasoline-fuelled internal combustion engines around $75 \%$ of the total energy is expelled to the environment as heat, thus, with the appropriate device, this waste energy can be reused. ${ }^{1,2}$

Thermoelectric generators (TEGs) are solid-state devices which can convert heat to electricity or vice versa based on the Seebeck or Peltier effects and are generally used for power generation or cooling applications, respectively. ${ }^{3,4}$ The efficiency of these devices is related to the dimensionless figure of merit $Z T=\frac{S^{2} \sigma T}{\left(k_{e}+k_{p}\right)}$, where $S$ corresponds to the Seebeck coefficient $\left(\mathrm{V} \mathrm{K}^{-1}\right) ; \quad \sigma$ is the electrical conductivity $\left(\mathrm{S} \mathrm{m}^{-1}\right) ; \mathrm{k}_{\mathrm{e}}$ and $\mathrm{k}_{\mathrm{p}}$ correspond to the electronic and phononic contribution of the thermal conductivity $\left(\mathrm{W} \mathrm{m}^{-1} \mathrm{~K}^{-1}\right)$, and $\mathrm{T}$ is the absolute temperature and $S^{2} \sigma$ represents the power factor (PF). The thermal lattice conductivity is given by $k_{l}=\Sigma_{m}(c v l)_{m}$, where $m$ is the heat carrier per mode, $v$ the speed of propagation, and $/$ the mean free path of the carrier. Weak bonding, high atomic mass and complex atomic structures govern the alteration of these atomic-level parameters. At the macroscale, the reduction of the propagation of lattice vibrations (phonons) is achieved through the scattering at grain boundaries. The realization of high-performance TE materials therefore requires a high ZT value, which in turn requires a large $S^{2} \sigma$ and a low $\mathrm{K}$. A high power factor can produce significantly more output power from better electrical and thermal impedance matching than optimization of ZT. ${ }^{5}$ This provides a significant materials challenge as it is hard to decouple these highly inter-dependent parameters. One of the most widely used materials is currently $\mathrm{Bi}_{2} \mathrm{Te}_{3}(\mathrm{ZT} \sim 1)$ whose TE properties can be modestly enhanced through appropriate nanoscale processing. ${ }^{6}$ Bismuth telluride has been at the core of commercial thermoelectric materials for low grade energy and compact cooling applications since the 1950s. A new material is needed to disrupt the sustainability issues associated with Tellurium.

TE power generation has the potential of becoming a transformative technology for renewable energy generation, provided that the low efficiency of current TE materials can be addressed by developing novel materials with high ZT. Metal organic frameworks (MOFs) could be good candidates for new TE materials given their relatively low thermal conductivity (approx. 0.1-0.5 times that of $\mathrm{Bi}_{2} \mathrm{Te}_{3}$ ) and tuneable electrical conductivity.

MOF are porous hybrid materials composed of inorganic and organic building blocks, in which metal centres or clusters are coordinated to organic linkers. ${ }^{7}$ The most attractive characteristic of these materials is the capability to modify their pore size, shape and topology, which depends on the 
selected building blocks. Furthermore, inherent porosity of these materials is a feature that can reduce the phonon mean free path leading to a lower thermal conductivity. ${ }^{8}$ Due to their structural and chemical tunability, MOFs have been investigated for technological applications such as gas separation ${ }^{9}$, catalysis ${ }^{10,11}$, biomedicine ${ }^{12}$ and energy applications. ${ }^{13,14}$

The research of MOFs as potential thermoelectric materials is still in its infancy, particularly due to their poor electrical properties. However, a targeted molecular design of this material with better charge transport properties can be achieved due to a wide variety of choices for metal and organic building units. Most MOFs are intrinsically insulating because there are no delocalized electronic states derived from the lack of overlap between the metal and ligand orbitals, although the heterogeneity in atomic species and ligands in MOFs could lead to a predictably low $k$, a fundamental property for high efficiency in thermoelectric materials. ${ }^{15}$ Post-synthetic approaches such as the introduction of redox active guest organic molecules within the pores of insulating MOFs such as HKUST-1 $\left(\mathrm{Cu}_{3}(\mathrm{BTC})_{2}\right.$, where $\mathrm{BTC}=1,3,5$-benzenetricarboxylate) have been reported to significantly improve their electrical conductivity ${ }^{16}$ Another strategy to build semiconducting MOF architectures involves the introduction of electroactive or highly conjugated ligands, which enhances the long-range charge delocalization and a number of semiconducting MOFs have been developed through this approach. ${ }^{17,18}$ For a significant up to date review about design strategies and charge transport mechanisms in conductive MOFs, the reader may consult the work reported by Xie et al. ${ }^{19}$

In 2012, Hmadeh and co-workers ${ }^{20}$ reported the first synthesis of metal catecholate $M_{3}(H H T P)_{2}$ frameworks (where $\mathrm{M}=\mathrm{Ni}(\mathrm{II}), \mathrm{Co}(\mathrm{II}), \mathrm{Cu}(\mathrm{II})$ and $\mathrm{HHTP}=2,3,5,6,10,11$-hexahydroxytriphenylene) in order to assess them as energy storage materials. The architecture of these materials displays a honeycomb structure according to high-resolution transmission electron microscopy (HRTEM) images. $\mathrm{Cu}_{3}(\mathrm{HHTP})_{2}$ is a semiconducting MOF comprising coordinating $\mathrm{Cu}$ ions with a 2,3,6,7,10,11hexahydroxytriphenylene (HHTP) ligand leading to a 2D hexagonal lattice (fig. 1) with a honeycomblike porous structure along the c-axis, similar to that observed in graphene. The distance between the stacking layers is $3.3 \AA$ A and the pore size is $\sim 18 \AA \AA^{21}{ }^{21}$ The electrical conductivity of $\mathrm{Cu}_{3}(\mathrm{HHTP})_{2}$ single crystals has been reported to be as high as $0.2 \mathrm{~S} \mathrm{~cm}^{-1}$, which is currently among the best conducting MOFs. As a consequence of this inherent conductivity, much of the research conducted on $\mathrm{Cu}_{3}(\mathrm{HHTP})_{2}$ has focussed on chemiresistive sensing and energy storage applications. ${ }^{15-34}$ (Table SI1) 


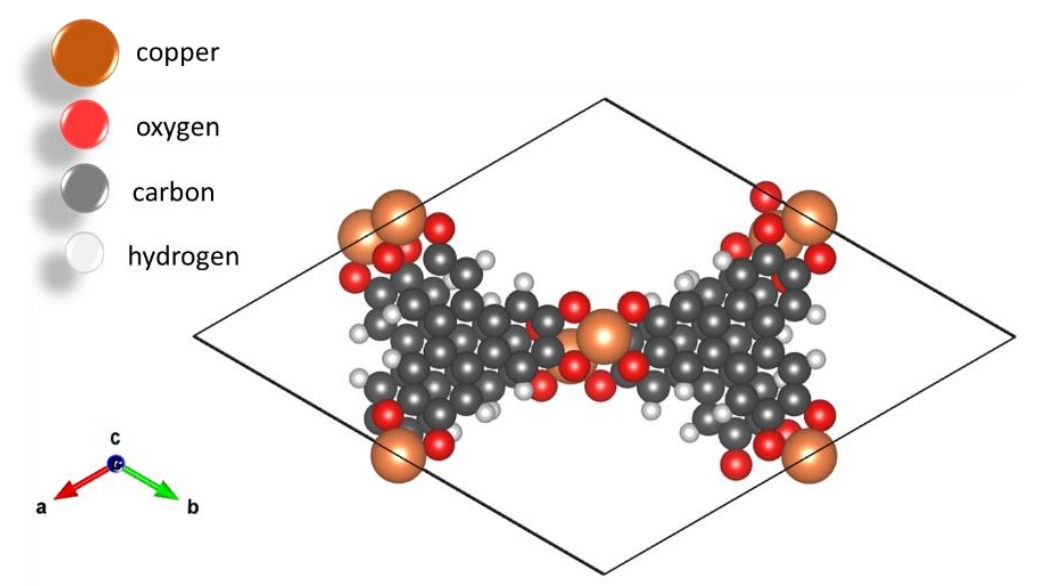

Figure 1. Crystal structure of $\mathrm{CU}_{3}(\mathrm{HHTP})_{2}$ displaying a slipped-parallel (AB) packing mode. (c-axis pointing out of the plane of the image)

Investigations on conductive MOFs as potential TEG materials have this far been limited to $7,7,8,8$ tetracyanoquinodimethane infiltrated HKUST-1 (TCNQ@ $\left.\mathrm{Cu}_{3}(\mathrm{BTC})_{2}\right)$ thin films and $\mathrm{Ni}_{3}(2,3,6,7,10,11$ hexaiminotriphenylene $)_{2}\left(\mathrm{Ni}_{3}[\mathrm{HITP}]_{2}\right)$ pellets exhibiting figures of merit of $7 \times 10^{-5}$ and $1.19 \times 10^{-3}$ at 298 $\mathrm{K}$, respectively (Table S2). ${ }^{35-37}$ These values are comparable to other classes of thermoelectric materials such as nanostructured materials $\left(\mathrm{Bi}_{2} \mathrm{~S}_{3}\right.$ nanowires $\left.{ }^{38}\right)$, metal oxides $\left(\mathrm{Ca}_{0.8} \mathrm{Dy}_{0.2} \mathrm{MnO}_{3}{ }^{39}\right)$ and conducting polymers (poly(3,4-ethylenedioxythiophene):polystyrenesulfonate/polypyrrole/paper ${ }^{40}$ ). (refer to fig. S1)

A current challenge to enable the technological implementation of MOFs is the processing of these materials onto solid supports since MOFs are routinely prepared as powders. The fabrication of MOFs as thin films is a promising strategy to incorporate these materials into robust devices, providing higher stability and unique properties derived from the interaction between the MOFs and the substrate surface. ${ }^{41,42}$ The successful application of MOF thin films in thermoelectric and other devices ideally requires a cost-effective and high throughput fabrication process where the crystallinity of the framework is preserved. Compared to other deposition methods (e.g. chemical vapour deposition (CVD), hydrothermal process), the advantages of electrodeposition include room temperature film formation without the necessity of applying a vacuum, shorter synthesis times, low-cost equipment and easy scalability. ${ }^{43}$ In fact, nowadays the large-scale production of MOFs can be conducted through electrochemical routes. ${ }^{44}$

The electrochemical synthesis of MOFs can be classified into two approaches: direct and indirect electrosynthesis. The strengths and shortcomings of these approaches have been discussed elsewhere. ${ }^{45}$ The former is based on the nucleation of MOF crystals directly on the electrode surface by a one-step synthesis procedure. For instance, the anodic dissolution method relies on the supply of the metal ions by the oxidation of the corresponding metal of interest (e.g., Cu foils, Zn plates), 
which is immersed in an electrolyte containing the organic linker. Indirect electrochemical approaches refer to those methods where a series of steps (e.g. surface preparation, anchoring of a linker) are required to grow the desired MOF. These methods offer facile control over crystallite size, thickness and homogeneity of MOF films formed in-situ by modulating parameters such as voltage/current, temperature, $\mathrm{pH}$, electrolyte, concentration and typically reaction times are lower compared to traditional solvothermal synthesis. ${ }^{37,46}$

The electrochemical synthesis of MOFs is conducted via a two-electrode or three-electrode configuration, where the latter provides enhanced control over the potential and the monitoring of the current or vice versa. The oxidation rate of the metal can be controlled by regulating the voltage applied to the electrode. ${ }^{47}$ Furthermore, the formation of adherent and continuous thin films with controllable thicknesses over conducting substrates is possible using this technique. In addition, the growth of MOFs on transparent conducting substrates ${ }^{48}$ is a promising field for the electronics industry, as integrated, patterned or sensitive devices are not usually fabricated on pure metal substrates.

In this work, the electrochemical deposition of the semiconducting $\mathrm{MOF} \mathrm{Cu}_{3}(\mathrm{HHTP})_{2}$ has been carried out on different substrates including $\mathrm{Au} / \mathrm{SiO}_{2}$ and fluorine-tin oxide (FTO) and its potential thermoelectric properties are investigated for the first time by determining the Seebeck coefficient and electrical resistivity, and through calculation of the power factor. The power factor is directly related to the maximum power that can be delivered to a load and if the temperature drop across a material is small then output power is more important than efficiency. This is especially true for MOFs whose thermal conductivity is reported to be very low. ${ }^{49}$

\section{Results and Discussion}

\section{Hydrothermal synthesis of bulk $\mathrm{Cu}_{3}(\mathrm{HHTP})_{2}$}

Bulk phases of $\mathrm{Cu}_{3}(\mathrm{HHTP})_{2}$ were initially synthesised under hydrothermal conditions according to the experimental procedure reported by Hoppe et al. ${ }^{27}$ This involves the reaction of $\mathrm{Cu}\left(\mathrm{NO}_{3}\right)_{2}$ and the HHTP linker at $80^{\circ} \mathrm{C}$ for $24 \mathrm{hrs}$ in the presence of aqueous ammonium hydroxide as an additive to enhance the dissolution and deprotonation of the ligand. This approach allowed us to gather information about the crystallinity and thermal stability of $\mathrm{Cu}_{3}(\mathrm{HHTP})_{2}$ prior to electrochemical film deposition studies and thermoelectric characterisation. The crystallinity of the $\mathrm{Cu}_{3}(\mathrm{HHTP})_{2}$ bulk sample was confirmed by PXRD measurements (fig. 2-a). The diffraction pattern displays characteristic peaks at $2 \theta=4.95^{\circ}, 9.76^{\circ}$ and $12.82^{\circ}$ and $16.73^{\circ}$, corresponding to the (100), (200), (210) and (220) planes, respectively. The broad peak located at $2 \theta=28.84^{\circ}$ corresponds to the (001) plane and is generally related to the degree of disorder of the stacking layers along the crystallographic c-direction in 2D MOFs. ${ }^{50}$ The peak position and full width at half-maximum (FWHM) of the (001) reflection were 
extracted from a line broadening fit using a pseudo-Voigt function. The obtained values were employed in the Bragg equation to calculate the interlayer distance between the stacked $\mathrm{Cu}_{3}(\mathrm{HHTP})_{2}$ sheets giving a value of $3.10 \AA$ Á. The lattice parameters of bulk $\mathrm{Cu}_{3}(\mathrm{HHTP})_{2}$ were determined to be $\mathrm{a}=$

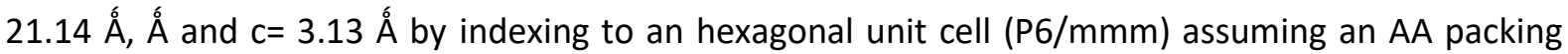
structure as a starting model. ${ }^{27}$ (fig. S2)

Thermogravimetric analysis (TGA) of $\mathrm{Cu}_{3}(\mathrm{HHTP})_{2}$ displays two distinct mass loss steps. (fig. 2-b) The first occurs in the temperature range from 30 to $120^{\circ} \mathrm{C}$ with a weight loss of $12.85 \%$ and is attributed to the desorption of water molecules present in the as-synthesised sample and any exchanged EtOH molecules arising from washing of the solid during work-up. The second mass loss step of $70.5 \%$ has an onset temperature of $230^{\circ} \mathrm{C}$ and corresponds to the thermal decomposition of the $\mathrm{Cu}_{3}(\mathrm{HHTP})_{2}$ framework resulting from ligand breakdown (theoretical 71.5\%), which is in good agreement with the reported stability. ${ }^{27,28}$ There is no further mass loss beyond this temperature indicating the complete decomposition of the $\mathrm{Cu}_{3}(\mathrm{HHTP})_{2}$ and the remaining $\mathrm{CuO}$ (29.5\%) after the TGA measurement under air is complete is in good agreement with that expected $(28.5 \%)$ from the framework formula of $\mathrm{Cu}_{3} \mathrm{C}_{36} \mathrm{H}_{18} \mathrm{O}_{12}$.

a)

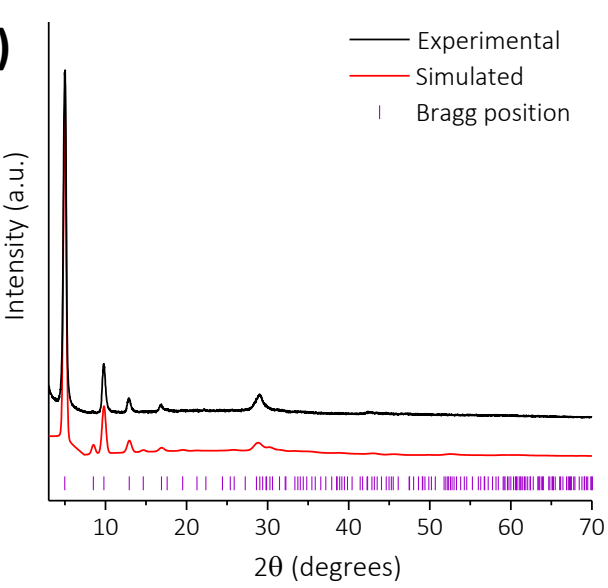

c)

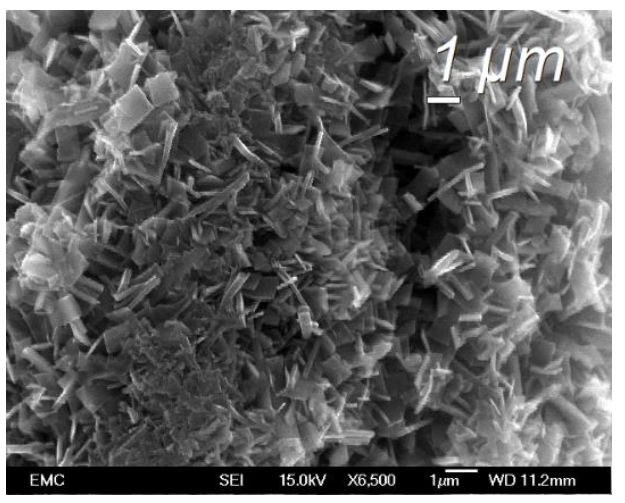

b)

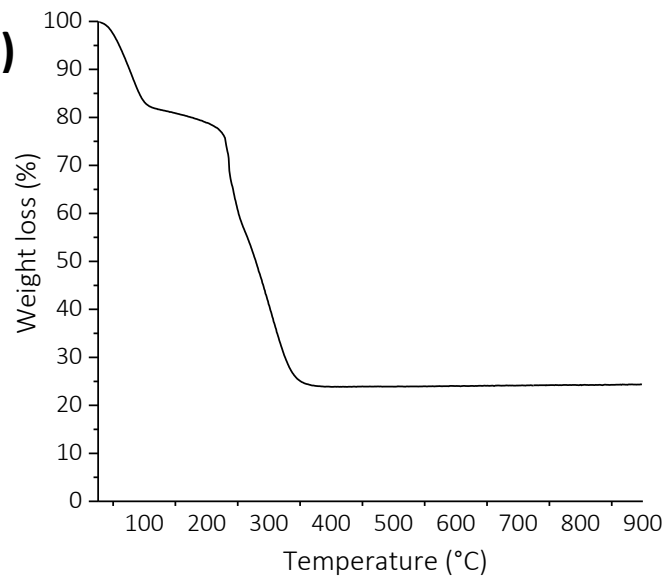

d)

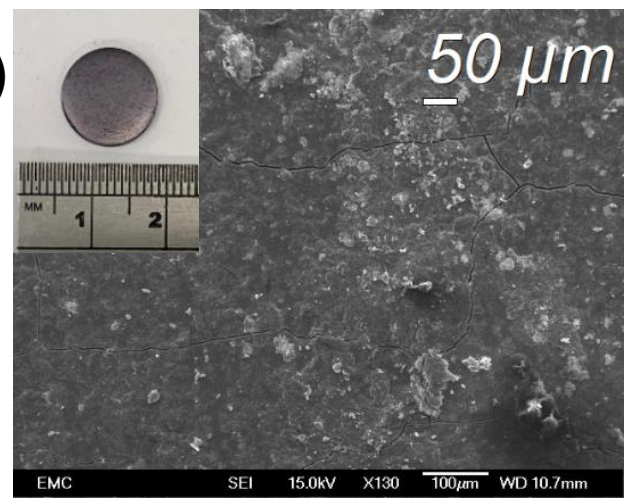

Figure 2. PXRD pattern $\left(R_{w}=5.62 \%, R_{p}=4.34 \%\right)$ a) and TGA curve b) of $\mathrm{Cu}_{3}(H H T P)_{2}$. SEM images of $\left.\mathrm{Cu}_{3}(H H T P)_{2} \mathrm{C}\right)$ following hydrothermal synthesis and prepared as a drop casted powder and d) the surface and photograph of the pressed pellet (inset). 
The morphology of the $\mathrm{CU}_{3}(\mathrm{HHTP})_{2}$ powder was investigated by scanning electron microscopy (SEM) (fig. 2-c; fig. S3). Homogeneous randomly oriented plate-like particles with sizes of $\sim 700 \mathrm{~nm}$ are observed, and the thickness of the platelets are estimated to be $190 \mathrm{~nm}$.

Following characterisation of the bulk $\mathrm{Cu}_{3}(\mathrm{HHTP})_{2}$ powder, pellets of the framework $(12.7 \mathrm{~mm} \varnothing \times 0.8$ $\mathrm{mm}$ ) were prepared for TE measurements using a hydraulic press (15 minutes at an applied weight of 10 ton). The internal microstructure and compactness of the $\mathrm{Cu}_{3}(\mathrm{HHTP})_{2}$ pellet was investigated by SEM (fig. 2-d). Top view images show a high densification of the sample as expected and the grain boundaries are also visible. Cross-sectional images show similar densification along the transversal axis of the pellet, although some cracking is also observed most likely arising from the splitting of the pellet for charactersation. Higher magnification images revealed that despite the grain boundaries observed, a good overall interconnection between the crystals is achieved by the pelletization process (fig. S4)

\section{Thermoelectric measurements of $\mathrm{Cu}_{3}(\mathrm{HHTP})_{2}$ pressed pellets}

Thermoelectric measurements were conducted at ambient conditions using a home-built system (fig. S5), which consists of four contact probes and two Peltier modules. The Seebeck coefficient was determined by applying a temperature differential across the sample while measuring the voltage drop with two probes, each one located at the hot and cold sides of the sample, respectively. At the same time, the temperature difference was monitored with two thermocouples, each one located at the hot and cold sides of the sample. Indium contacts were placed on top of the $\mathrm{Cu}_{3}(\mathrm{HHTP})_{2}$ pellet in order to improve the electrical contact between the sample and the instrument probes. Good ohmic contact is demonstrated by the linear response observed in the I-V curves (fig. S6).

Figure 3-a shows the measured Seebeck voltage as a function of the temperature difference applied between the hot and cold side of the pressed $\mathrm{Cu}_{3}(\mathrm{HHTP})_{2}$ pellet. The Seebeck coefficient can then be determined as the gradient of the Seebeck voltage plotted against the temperature difference. $A$ Seebeck coefficient of $-7.24 \mu \mathrm{V} \mathrm{K}^{-1}$ was estimated from the slope of the linear fit (red line). The negative sign of the Seebeck coefficient indicates that the majority of charge carriers present in the $\mathrm{Cu}_{3}(\mathrm{HHTP})_{2}$ bulk pellet are electrons, suggesting that this MOF behaves as an $\mathrm{n}$-type material, which is a new finding. According to previous reports $\mathrm{Cu}_{3}(\mathrm{HHTP})_{2}$ has been identified as a p-type semiconductor as determined by ultraviolet photoelectron spectroscopy (UPV) that showed an increase in the Fermi level by $1.13 \mathrm{eV}$ after adsorption of $\mathrm{NH}_{3}{ }^{25}$ and in field effect transistor (FET) devices that demonstrated an increase in the channel current with decreasing gate voltage. ${ }^{26}$ In both 
studies the measurements were conducted on devices made with $10 \mathrm{~nm}$ thick $\mathrm{Cu}_{3}(\mathrm{HHTP})_{2}$ films. It is noteworthy that in the FET study an excess of oxygen was detected by X-ray photoelectron spectroscopy (XPS) inside the pores, which has been mainly attributed to solvent molecules. We believe that the reason for the difference in charge transport observed in our $\mathrm{Cu}_{3}(\mathrm{HHTP})_{2}$ material and that reported in the literature (i.e. $n$ - versus p-type conduction) may indeed be attributable to the presence of solvent molecules inside the pores and/or the adsorption of gases either within the pores or on the surface. However clearly more experimentation is required on this aspect which is currently ongoing as part of a companion study. Hall effect measurements on the $\mathrm{Cu}_{3}(\mathrm{HHTP})_{2}$ pellet resulted in a bulk carrier concentration of $2.2 \times 10^{17} \mathrm{~cm}^{-3}$.

Figure 3-b shows the electrical conductivity of the $\mathrm{Cu}_{3}(\mathrm{HHTP})_{2}$ pellet measured as a function of temperature. The electrical conductivity of the $\mathrm{Cu}_{3}(\mathrm{HHTP})_{2}$ pellet measured at $301 \mathrm{~K}$ is $\sigma=3.80 \times 10^{-3}$ $\mathrm{S} \mathrm{cm}{ }^{-1}$, which is comparable to conductivities of $\mathrm{Cu}_{3}(\mathrm{HHTP})_{2}$ in bulk reported under vacuum conditions. ${ }^{27}$ (also table SI1) Additional electrical measurements were conducted between 301 and $316 \mathrm{~K}$ at ambient conditions where the semiconducting behaviour of $\mathrm{Cu}_{3}(\mathrm{HHTP})_{2}$ was confirmed since an increase in conductivity upon increasing the temperature was observed (fig. 3-b). Further, the band gap of the pellet was calculated to be $2.68 \mathrm{eV}$ from an Arrhenius plot (fig. S7) which is consistent with the somewhat resistive nature of the material. According to our literature survey, thermoelectric measurements on bare bulk MOFs have only been conducted on $\mathrm{Ni}_{3}(\mathrm{HITP})_{2}$. The calculated power factor of bulk $\mathrm{Cu}_{3}(\mathrm{HHTP})_{2}$ is $2 \times 10^{-5} \mu \mathrm{W} \mathrm{m}^{-1} \mathrm{~K}^{-2}$. This value is four orders of magnitude lower compared to $\mathrm{Ni}_{3}(\mathrm{HITP})_{2}\left(\mathrm{PF}=0.832 \mu \mathrm{W} \mathrm{m}{ }^{-1} \mathrm{~K}^{-2}\right.$, pressed pellet) ${ }^{37}$, and while we largely attribute this to the much lower electrical conductivity of $\mathrm{Cu}_{3}(\mathrm{HHTP})_{2}$ the effect of cracks in the material cannot be entirely ruled out.

a)

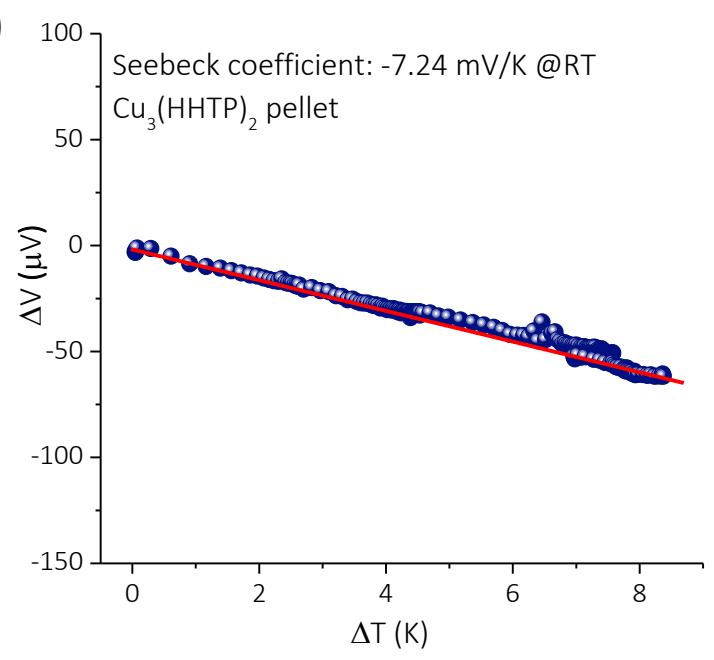

b)

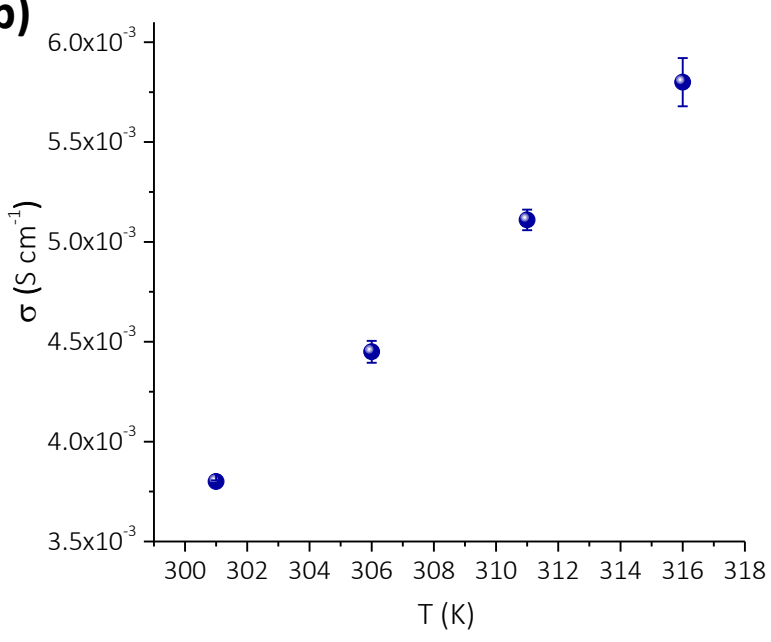

Figure 3. a) Seebeck voltage versus temperature difference and b) electrical conductivity as a function of temperature for $\mathrm{Cu}_{3}(\mathrm{HHTP})_{2}$ pressed pellet. Standard error was calculated from three repeat measurements. 


\section{Anodic Electrosynthesis of $\mathrm{Cu}_{3}(\mathrm{HHTP})_{2}$ onto Transparent Conducting Substrates}

$\mathrm{Cu}_{3}(\mathrm{HHTP})_{2}$ thin films have been previously produced from mother solutions ${ }^{23}$, layer-by-layer deposition ${ }^{25}$, growth at liquid-liquid interfaces ${ }^{26}$ and by spray coating ${ }^{27}$ methods. In this work we extend film preparation to include electrochemical synthesis as a versatile method to grow $\mathrm{Cu}_{3}(\mathrm{HHTP})_{2}$ initially on transparent conducting substrates by anodization ${ }^{43}$ for potential applications as backcontact electrodes in the optoelectronics field. ${ }^{51} \mathrm{~A}$ metallic copper layer was first deposited onto FTO and then oxidised to generate the metal ions to be coordinated to the framework-forming HHTP ligands (fig. S8). The copper film was electrodeposited at a potential of $-0.8 \mathrm{~V}$ vs SCE for $1 \mathrm{hr}{ }^{46}$ It was then immersed in a solution containing the HHTP ligand at a range of anodic potentials $(+0.435,+0.5$ $\mathrm{V},+0.6 \mathrm{~V}$ and $+0.7 \mathrm{~V}$ vs SCE) with tributylmethylammonium methyl sulfate (MTBS) as the supporting electrolyte.

Figure 4 shows the grazing-incidence $x$-ray diffraction (GIXRD) patterns of the electrodeposited $\mathrm{Cu}_{3}(\mathrm{HHTP})_{2}$ at different applied potentials onto an FTO glass substrate. Reflections located at $\sim 4.86$, 9.6, 12.7 and $\sim 28$ degrees $2 \theta$ correspond to the MOF diffraction peaks for the (100), (200), (210) and (002) planes, respectively, as previously observed for the bulk framework.

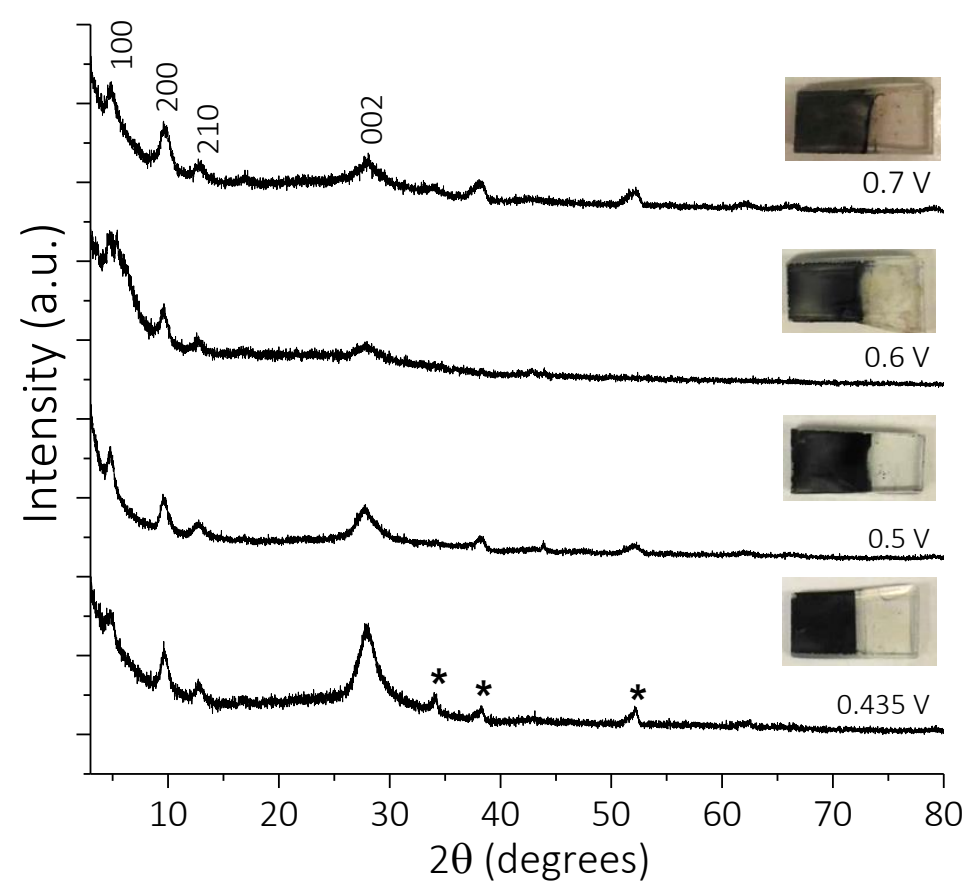

Figure 4. GIXRD of electrodeposited $\mathrm{CU}_{3}(\mathrm{HHTP})_{2}$ thin films onto FTO substrate a) at different applied potentials and their corresponding photographs (insets). $\left({ }^{*}\right)$ corresponds to FTO glass diffraction peaks. 
As the potential is increased the crystallinity of the MOF appears to decrease (Table S3) which may be due to the faster oxidation rate at a higher potential allowing insufficient time to coordinate to the supplied ligand in solution. We note that there was no significant homogeneous MOF formation in the solution and most was deposited onto the substrate. Further, no preferred orientation was observed for any of the deposited films. Nonetheless, a significant drop in intensity in the peak corresponding to the (002) plane at $2 \theta \sim 27.91$ was noted. This finding suggests that higher applied potentials may lead to increased disorder along the stacking axis in the layered structure of electrodeposited $\mathrm{Cu}_{3}(\mathrm{HHTP})_{2}$ films. It is important to preserve the crystallinity along this crystallographic direction, since one of the mechanisms of charge transport present in conductive MOFs, namely through-space, occurs via $\pi$-stacked aromatic ligands in close proximity ( 3.5 Á). ${ }^{18}$ Therefore, a long-range ordering and shorter inter-ligand stacking allows a better orbital overlap among the ligands enhancing the overall charge mobility.

The crystal (domain) size of every sample was calculated from experimental GIXRD patterns giving

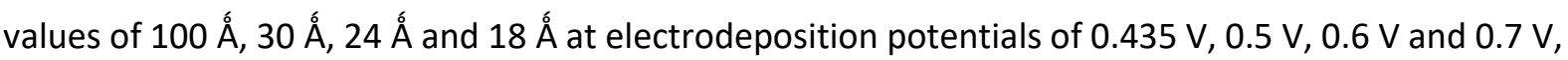
respectively. The decrease in crystal size as the potential is increased has previously been observed in the electrosynthesis of ZIF- $8^{52}$ and HKUST- ${ }^{43}$, and has been explained in terms of nucleation theory since a higher potential increases the concentration of metal ions near the surface electrode. The calculated interlayer distance of the electrodeposited $\mathrm{Cu}_{3}(\mathrm{HHTP})_{2}$ at $0.435 \mathrm{~V}$ is $3.17 \AA$, which is in good agreement with the reported values in the literature and for the bulk material.

By visual inspection, the applied potentials also had an impact on the continuous coverage of the films (fig. 4, insets). As the potential was increased, the coverage of the samples becomes less homogenous. The morphology and thickness of the electrosynthesised $\mathrm{Cu}_{3}(\mathrm{HHTP})_{2}$ films on FTO substrates were characterised by SEM (fig. 5). The $\mathrm{Cu}_{3}(\mathrm{HHTP})_{2}$ film deposited at $0.435 \mathrm{~V}$ consists of compact randomly oriented particles with an approximately spherical shape, and film thickness is ca. $4 \mu \mathrm{m}$ (fig. 5-a). Electrodeposition conducted at $0.5 \mathrm{~V}$ (fig. 5-b) shows a more uneven film comprised of semi-spherical shaped particles. The estimated thickness of the $\mathrm{Cu}_{3}(\mathrm{HHTP})_{2}-0.5 \mathrm{~V}$ from SEM images ranges from 3.8 to $6.8 \mu \mathrm{m}$. $\mathrm{Cu}_{3}(\mathrm{HHTP})_{2}$ film electrodeposited at $0.6 \mathrm{~V}$. (fig. 5 -c) shows irregularly shaped particles, and the thickness of the $\mathrm{Cu}_{3}(\mathrm{HHTP})_{2}-0.6 \mathrm{~V}$ is $\sim 7 \mu \mathrm{m}$. An applied potential of $0.7 \mathrm{~V}$ leads to larger particles with irregular shapes. The estimated thickness of this MOF film is $~ 6.5 \mu \mathrm{m}$ (fig. $5-\mathrm{d}$ ), which is consistent with that observed at $0.6 \mathrm{~V}$.

These findings suggest that by applying an appropriate potential, it is possible to control the morphology and decrease the interstitial gaps between the intergrown MOF microcrystals due to the coalescence of the formed particles leading to more dense films. ${ }^{43}$ 

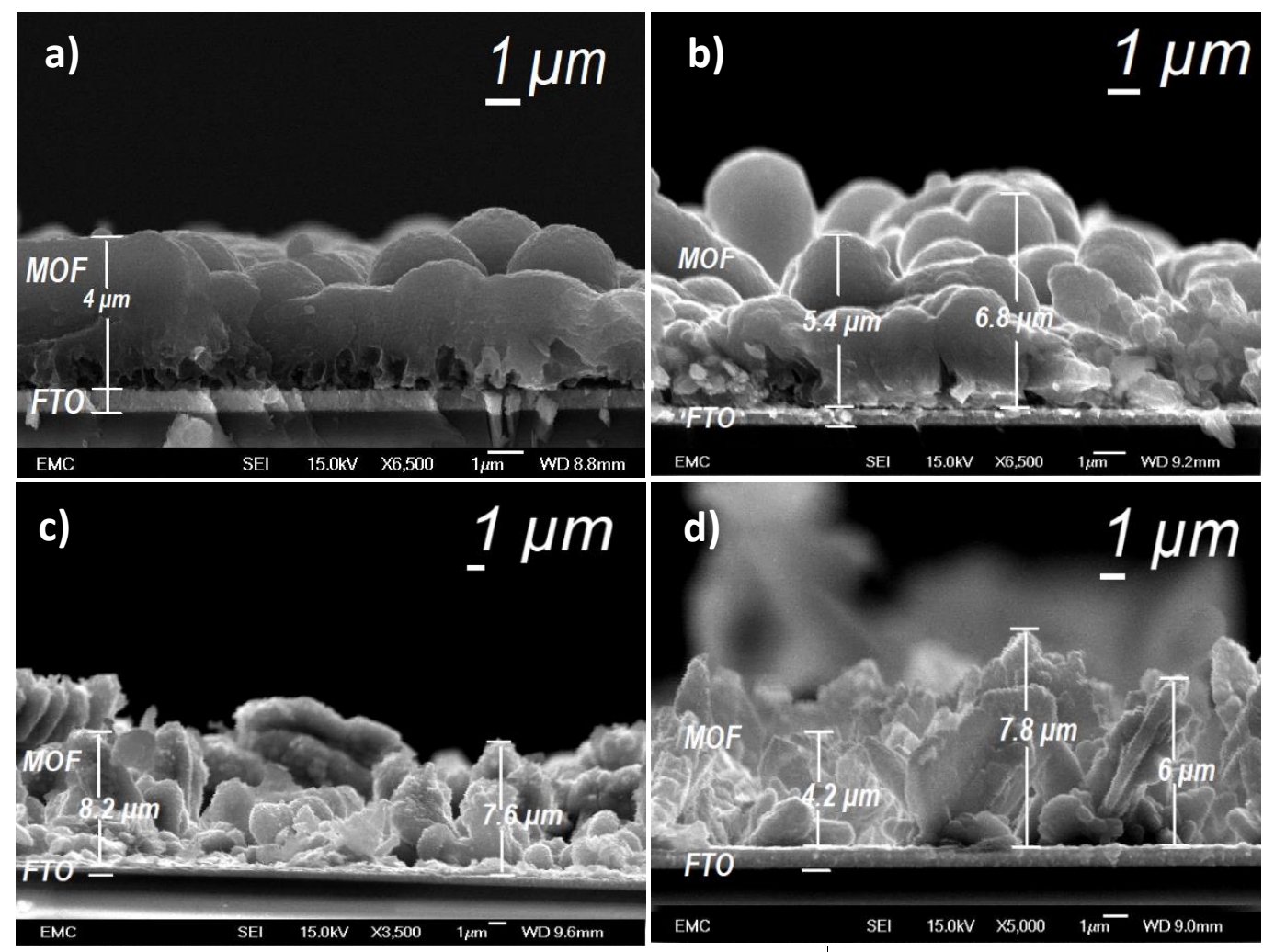

Figure 5. SEM cross section images of electrodeposited $\left.\mathrm{Cu}_{3}(\mathrm{HHTP})\right|_{2}$ films onto FTO at different applied potentials. a) $0.435 \mathrm{~V}$, b) $0.5 \mathrm{~V}, \mathrm{c}) 0.6 \mathrm{~V}$ and d) $0.7 \mathrm{~V}$.

The electrical characterisation of electrodeposited semiconductor materials is to some extent challenging since the sample must be detached from the conducting substrate to avoid its contribution to the measurements. Initially, detaching the electrodeposited $\mathrm{Cu}_{3}(\mathrm{HHTP})_{2}$ from FTO glass was attempted by using a solution of polymethylmethacrylate (PMMA) in cholorobenzene as a transfer agent. (SI-Methods) However, due to the strong adhesion of the MOF film to the FTO substrate the transfer could not be effectively achieved. For this reason, the electrodeposition of $\mathrm{Cu}_{3}(\mathrm{HHTP})_{2}$ on a second conducting substrate $\left(\mathrm{Au} / \mathrm{SiO}_{2}\right)$ was investigated.

\section{Anodic Electrosynthesis of $\mathrm{Cu}_{3}(\mathrm{HHTP})_{2}$ onto $\mathrm{Au} / \mathrm{SiO}_{2}$ substrates}

The $\mathrm{Cu}_{3}(\mathrm{HHTP})_{2}$ thin films in this work were electrochemically deposited onto $\mathrm{Au} / \mathrm{SiO}_{2}$ substrates that were pre-covered in an overlayer of $\mathrm{Cu}$ metal. Cyclic voltammetry of the $\mathrm{Au} / \mathrm{SiO}_{2}$ substrate immersed in a solution containing $0.01 \mathrm{M} \mathrm{Cu}(\mathrm{SO})_{4}$ and $0.1 \mathrm{M} \mathrm{KCl}$ was employed to determine the optimum deposition conditions for the metallic Cu layer (fig. S9). From the reverse scan, a reduction peak with a maximum current of $-0.240 \mathrm{~V}$ vs SCE was observed, indicating that the reduction of copper occurs in one step involving a two-electron transfer. In the anodic region, two oxidation peaks are located at $+0.214 \mathrm{~V}$ and $+0.362 \mathrm{~V}$ (vS SCE), which corresponds to the electrochemical conversion of $\mathrm{Cu}^{0} \rightarrow \mathrm{Cu}+{ }^{1}$ 
and $\mathrm{Cu}^{+1} \rightarrow \mathrm{Cu}^{+2}$, respectively. ${ }^{53}$ The electrodeposition of the copper layer was thus carried out using an applied potential of $-0.270 \mathrm{~V}$ for 60 minutes.

The formation of the $\mathrm{Cu}_{3}(\mathrm{HHTP})_{2}$ thin film was conducted through the anodic dissolution of the copper layer on the $\mathrm{Au} / \mathrm{SiO}_{2}$ substrate in a solution containing the HHTP ligand by applying a potential of $0.435 \mathrm{~V}$ for 120 minutes. These conditions were selected based on the prior optimum deposition conditions determined using the FTO substrate.

After deposition, the formation of a dark film displaying the characteristic blue colour of the $\mathrm{Cu}_{3}(\mathrm{HHTP})_{2}$ framework was observed. Once the $\mathrm{Cu}_{3}(\mathrm{HHTP})_{2}$ thin film was dried in air at ambient temperature, the MOF film was covered with a suspension of PMMA in cholorobenzene as previously described. The PMMA-MOF thin film samples were subjected to a drying process at $40^{\circ} \mathrm{C}$ for 12 hours. The $\mathrm{Cu}_{3}(\mathrm{HHTP})_{2}$ thin films were subsequently transferred intact from the $\mathrm{Au} / \mathrm{SiO}_{2}$ substrate by peeling away the PMMA which adheres sufficiently to the $\mathrm{Cu}_{3}(\mathrm{HHTP})_{2}$ film as outlined in fig. 6 . This is a necessary step to configure the films for thermoelectric measurements to remove the electrical contribution of the conducting substrate.

\section{Electrochemical synthesis}

Step 1.

Electrodeposition of $\mathrm{Cu}$

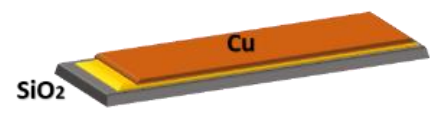

Step 2.

Anodic dissolution of $\mathrm{Cu}$

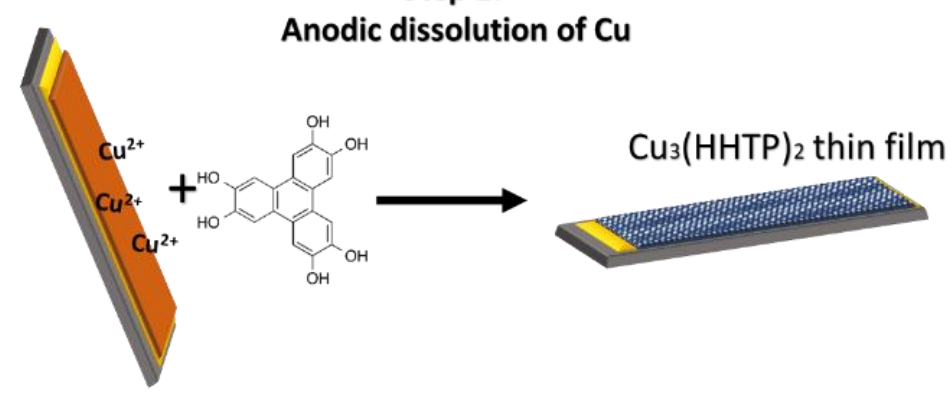

$\mathrm{Cu}_{3}(\mathrm{HHTP})_{2} @ P M M A$ film

Transfer method
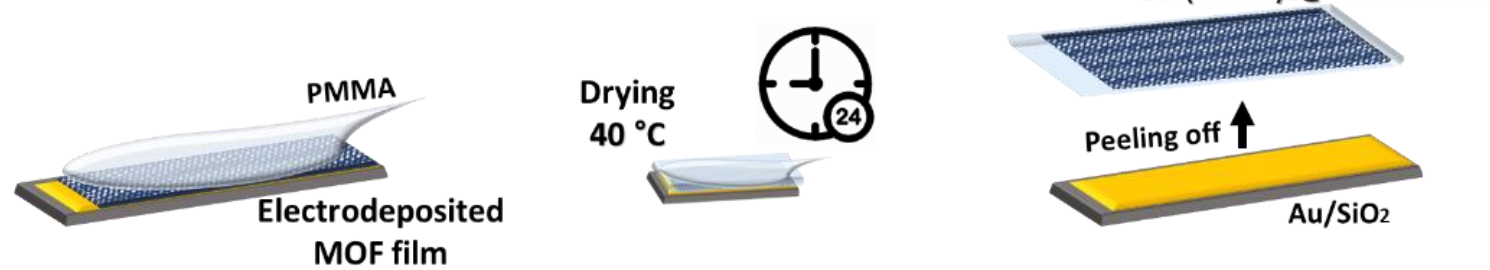

Figure 6. Schematic representation of the two-step anodic electrosynthesis of $\mathrm{Cu}_{3}(\mathrm{HHTP})_{2}$ and film transfer method. 
GIXRD patterns of the electrodeposited $\mathrm{Cu}_{3}(\mathrm{HHTP})_{2}$ on the $\mathrm{Au} / \mathrm{SiO}_{2}$ substrate and the transferred MOF film with PMMA are shown in fig 7. The successful electrodeposition and transfer of the MOF film was confirmed by the presence of sharp diffraction peaks located at $2 \theta=4.79^{\circ}, 9.56^{\circ}, 12.59^{\circ}$ and $27.86^{\circ}$, corresponding to the 100, 200, 210 and 002 planes, respectively. This is in excellent agreement with the bulk powder samples of $\mathrm{Cu}_{3}(\mathrm{HHTP})_{2}$, and in the transferred/removed sample the large broad peaobserved at $13^{\circ}$ is attributed to the amorphous nature of the PMMA support. ${ }^{54}$

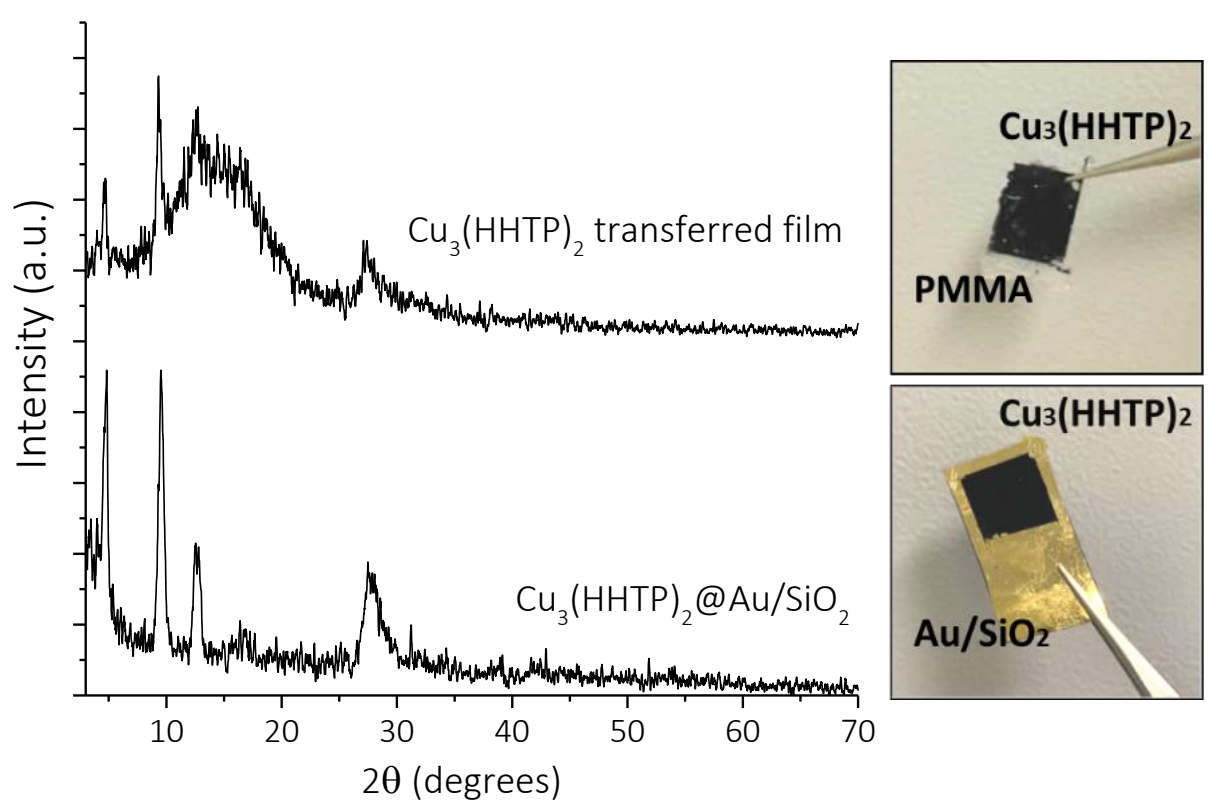

Figure 7. GIXRD patterns of the electrodeposited $\mathrm{Cu}_{3}(\mathrm{HHTP})_{2}$ onto $\mathrm{Au} / \mathrm{SiO}_{2}$ and $\mathrm{Cu}_{3}(\mathrm{HHTP})_{2}$ transferred film with their respective optical images.

The morphology of the electrodeposited $\mathrm{Cu}_{3}(\mathrm{HHTP})_{2}$ thin films was investigated by SEM (fig. 8-a,b,c). Spherical particles in a stacked-fashion array with an average diameter of $\sim 6 \mu \mathrm{m}$ were observed (fig. 8-a). Higher magnification images revealed that each of the spherical architectures is formed by $\mathrm{Cu}_{3}(\mathrm{HHTP})_{2}$ nanorods that are $\sim 85 \mathrm{~nm}$ in size. (fig. 8-b,c) From cross-sectional SEM measurements, a $\mathrm{CU}_{3}(\mathrm{HHTP})_{2}$ film thickness of $\sim 5 \mu \mathrm{m}$ was determined (fig. 8-d). The nanostructured morphology of the electrodeposited $\mathrm{Cu}_{3}(\mathrm{HHTP})_{2}$ thin films is a promising finding since this feature has previously been employed as a strategy to lower the thermal conductivity of materials by increasing their phonon scattering. ${ }^{55}$ This further suggests that electrodeposition of conducting MOFs could be a general strategy to prepare, structure and potentially optimise MOF thin films for electrical measurements and applications. 

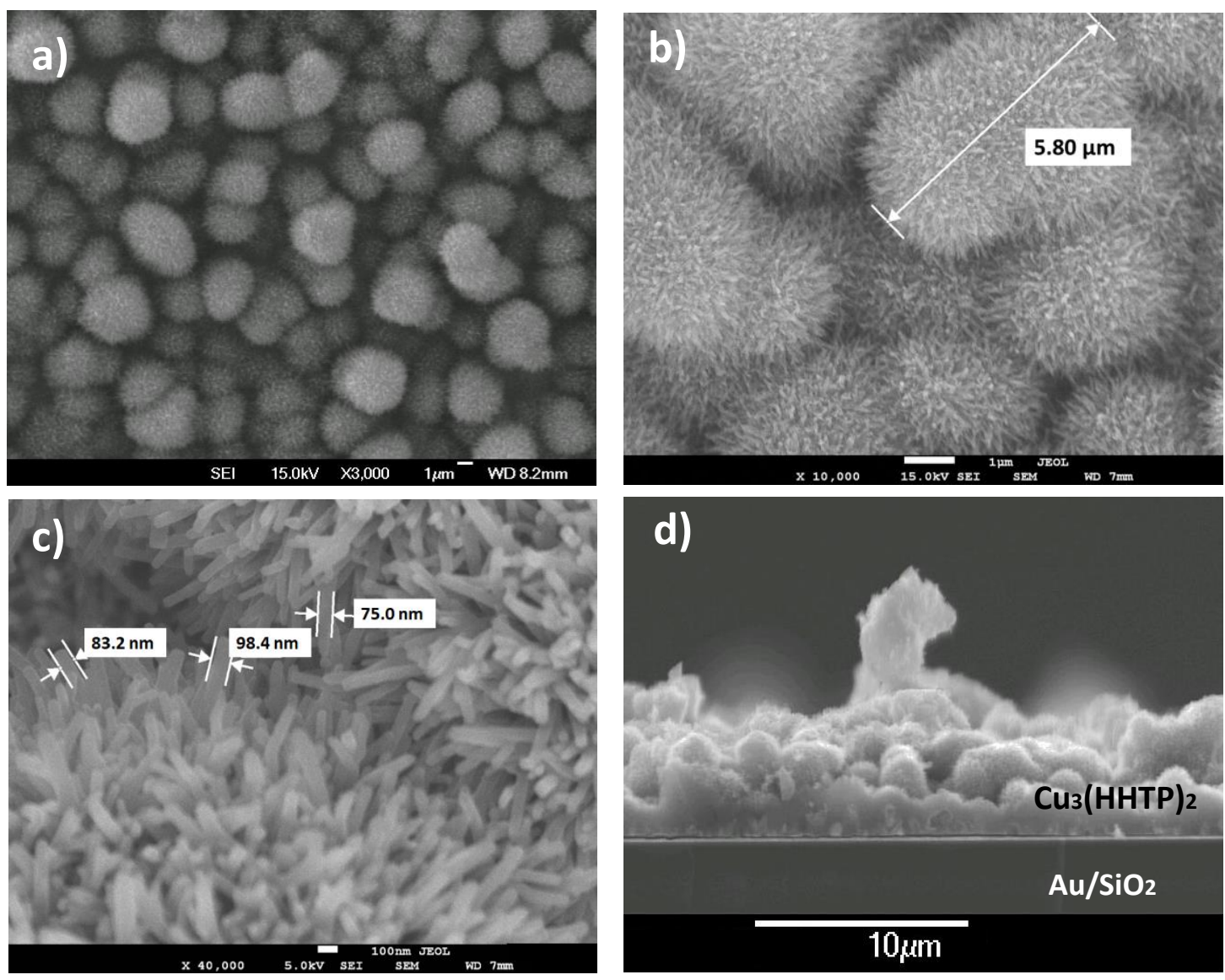

Figure 8. SEM images of electrodeposited $\mathrm{Cu}_{3}(\mathrm{HHTP})_{2}$ thin film onto $\mathrm{Au} / \mathrm{SiO}_{2}$ substrate. Top view $(a, b, c)$ showing the nanostructured morphology of the film and d) cross section view for thickness estimation.

\section{Thermoelectric measurements of electrodeposited $\mathrm{Cu}_{3}(\mathrm{HHTP})_{2}$ films}

Current-voltage (I-V) measurements were conducted to evaluate the quality of the electrical contacts between the PMMA transferred $\mathrm{Cu}_{3}(\mathrm{HHTP})_{2}$ thin films and the probes of the instrument. $A$ characteristic I-V linear curve indicating Ohmic behaviour was observed (fig. S6). This response suggests that despite the interstitial voids observed between the spherical particles of the MOF observed by SEM, the presence of the highly aggregated nanorods allows a good physical contact between the particles that potentially provides a favourable path for electron charge carriers. ${ }^{56}$ Furthermore, these measurements demonstrate that the transfer method of $\mathrm{Cu}_{3}(\mathrm{HHTP})_{2}$ thin films using PMMA as a transfer agent does not cause any significant cracking of the films as verified by SEM images (fig. S10). The flexible nature of the PMMA-MOF films makes these challenging to image in cross-section, but such images were successfully obtained following film removal with epoxy resin which suitably rigidified the films. These images (fig. S10) revealed the cross-section to be $\sim 5 \mu \mathrm{m}$ 
which is fully consistent with that of the as-deposited $\mathrm{Cu}_{3}(\mathrm{HHTP})_{2}$ films on the $\mathrm{Au} / \mathrm{SiO}_{2}$ substrate (fig. 8).

A current challenge in designing efficient thermoelectric materials relies on the interdependence of the variables involved to achieve a high figure of merit with respect to the charge carrier concentration. ${ }^{57}$ The increase in carrier concentration leads to an increase in the electrical conductivity of the material but because the contribution of free electrons in transporting heat energy is greater than the contribution generated by the lattice vibrations (phonons), the thermal conductivity is simultaneously increased. On the other hand, the increase in carrier concentration leads to low Seebeck coefficient values. Note that in-plane thermal conductivity of the $\mathrm{Cu}_{3}(\mathrm{HHTP})_{2}$ films (and pellets) reported in this work were not determined due to the difficulty in measuring this accurately given the demonstrable roughness of the samples. ${ }^{58-60}$
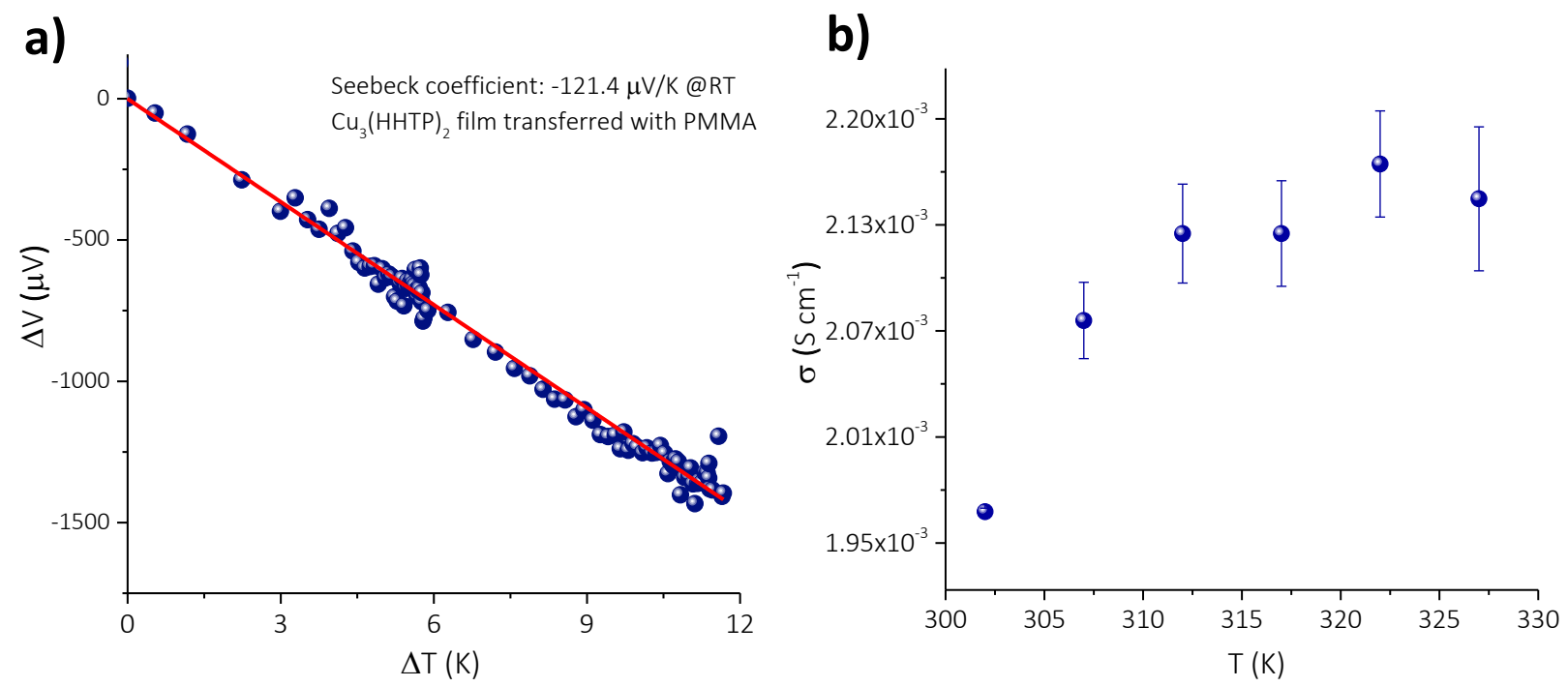

Figure 9. Thermoelectric characterization of the PMMA transferred $\mathrm{Cu}_{3}(\mathrm{HHTP})_{2}$ film: a) Seebeck voltage as a funcion of temperature difference and b) electrical conductivity as a function of the temperature. Standard error calculated from five repeats.

Fig. 9-a shows the Seebeck voltage as a function of temperature difference for the $\mathrm{Cu}_{3}(\mathrm{HHTP})_{2}$ thin film and from the gradient a Seebeck coefficient of $-121.4 \mu \mathrm{V} \mathrm{K}^{-1}$ could be determined, which differs significantly from values measured for Cu only films (fig. S11). This is the highest Seebeck coefficient that has been reported for this MOF so far and the negative sign is consistent with the n-type semiconducting behaviour determined for the pressed pellet.

As the Seebeck coefficient is inversely proportional to the charge carrier density in the material, Hall effect measurements were conducted to investigate the higher Seebeck coefficient observed in the MOF film compared to the pellet. The bulk charge carrier concentration of the PMMA-transferred 
$\mathrm{Cu}_{3}(\mathrm{HHTP})_{2}$ film was found to be $4.97 \times 10^{16} \mathrm{~cm}^{-3}$ which is 10 -fold lower than that determined for the MOF pellet. (Table S4) Clearly the lower Seebeck coefficient observed in the pellet may be explained in terms of the higher bulk charge carrier concentration. As revealed in fig. 9-b the electrical conductivity increases with temperature thereby confirming the semiconducting nature of the film. At $301 \mathrm{~K}$ the electrical conductivity has a value of $2.28 \times 10^{-3} \mathrm{~S} \mathrm{~cm}^{-1}$ which is slightly lower than that determined for the bulk pellet and may be attributable to PMMA residues left on the surface of the film during the transfer process. However this value still compares well with previously reported values in the literature on $\mathrm{Cu}_{3}(\mathrm{HHTP})_{2}$ films measured under vacuum conditions. (Table S1) A power factor of $3.15 \times 10^{-3} \mu \mathrm{W} \mathrm{m} \mathrm{m}^{-1} \mathrm{~K}^{-2}$ can be calculated for the $\mathrm{Cu}_{3}(\mathrm{HHTP})_{2}$ based on this data. The enhancement of the PF observed in the MOF film compared to the pellet arises (table S4) from the interdependence between the carrier density and the Seebeck coefficient variables. Our results demonstrate that the power factor can be tuned by nanostructuring of the films. This strategy has also been employed in order to lower the thermal conductivity in TE materials, however, the decrease of this property is usually accompanied by a reduction in the electrical conductivity.

\section{Conclusions}

In summary, the electrochemical deposition and thermoelectric properties including the Seebeck coefficient and power factor of the semiconducting 2-D MOF $\mathrm{Cu}_{3}(\mathrm{HHTP})_{2}$ are reported for the first time. Furthermore, electrochemical deposition is demonstrated as a promising and versatile method to grow MOFs, including on transparent conducting substrates potentially extending their applications to optoelectronics. Electrodeposited $\mathrm{Cu}_{3}(\mathrm{HHTP})_{2}$ thin films on $\mathrm{Au} / \mathrm{SiO}_{2}$ substrates were successfully transferred by a wet chemical method using a PMMA suspension. This approach led to a facile transfer of MOF thin films without compromising the crystallinity of the $\mathrm{Cu}_{3}(\mathrm{HHTP})_{2}$ framework as corroborated by GIXRD measurements. The semiconducting character of $\mathrm{Cu}_{3}(\mathrm{HHTP})_{2}$ in bulk and thin films was demonstrated by temperature-dependent electrical measurements, where a decrease in electrical resistivity with increasing temperature was observed. The electrochemical deposition led to the clean one-step formation of $\mathrm{Cu}_{3}(\mathrm{HHTP})_{2}$ thin films as observed in GIXRD measurements. Seebeck coefficient values for the $\mathrm{Cu}_{3}(\mathrm{HHTP})_{2}$ film are higher compared to the triphenylene-based MOF $\mathrm{Ni}_{3}(\mathrm{HITP})_{2}$. The study of semiconducting MOFs and their performance as TEG is expected to expand and offer alternatives to non-toxic, scalable and high-efficiency novel TEG materials.

\section{Acknowledgements}


M. Gonzalez-Juarez is supported by the National Council of Science and Technology-Mexico (CONACYT) under registry number 526108. MSMG acknowledges the project MAT2017-86450-C4-3-R and EF the financial support from the project 201950E057. We thank Dr Geoff Hyett for assistance with treatment of the bulk powder diffraction data. 


\section{References}

1 J. Yang and F. R. Stabler, J. Electron. Matter., 2009, 38, 1245-1251.

2 K. Zorbas, E. Hatzikraniotis and K. M. Paraskevopoulos, AIP Conf. Proc., 2010, 1203, 1137.

3 I. Nandhakumar, N. White and S. Beeby, Thermoelectric Materials and Devices, The Royal Society of Chemistry, Cambridge, 2017.

4 D. M. Rowe, Materials, Preparation, and Characterization in Thermoelectrics, CRC Press, Florida, 2012.

$5 \quad$ M. Wolf, R. Hinterding and A. Feldhoff, Entropy, 2019, 21, 1058.

6 I. T. Witting, T. C. Chasapis, F. Ricci, M. Peters, N. A. Heinz, G. Hautier and G. J. Snyder, Adv. Electron. Mater., 2019, 5, 1-20.

7 H. C. Zhou, J. R. Long and O. M. Yaghi, Chem. Rev., 2012, 112, 673-674.

8 D. S. Smith, A. Alzina, J. Bourret, B. Nait-Ali, F. Pennec, N. Tessier-Doyen, K. Otsu, H. Matsubara, P. Elser and U. T. Gonzenbach, J. Mater. Res., 2013, 28, 2260-2272.

9 H. S. Cho, J. J. Yang, X. Gong, Y. B. Zhang, K. Momma, B. M. Weckhuysen, H. X. Deng, J. K. Kang, O. M. Yaghi and O. Terasaki, Nat. Chem., 2019, 6, 562-570.

10 E. A. Dolgopolova, A. M. Rice, C. R. Martin and N. B. Shustova, Chem. Soc. Rev., 2018, 47, 4710-4728.

11 C. S. Diercks, Y. Liu, K. E. Cordova and O. M. Yaghi, Nat. Mater., 2018, 17, 301-307.

12 K. Lu, T. Aung, N. Guo, R. Weichselbaum and W. Lin, Adv. Mater., 2018, 30, 1-20.

13 J. G. Santaclara, F. Kapteijn, J. Gascon and M. A. Van Der Veen, CrystEngComm, 2017, 19, 4118-4125.

14 J. Zhou and B. Wang, Chem. Soc. Rev., 2017, 46, 6927-6945.

15 B. L. Huang, Z. Ni, A. Millward, A. J. H. McGaughey, C. Uher, M. Kaviany and O. Yaghi, Int. J. Heat Mass Transf., 2007, 50, 405-411.

16 A. A. Talin, A. Centrone, A. C. Ford, M. E. Foster, V. Stavila, P. Haney, R. A. Kinney, V. Szalai, F. El Gabaly, H. P. Yoon, F. Léonard and M. D. Allendorf, Science, 2014, 343, 66-69.

17 C. F. Leong, P. M. Usov and D. M. D’Alessandro, MRS Bull., 2016, 41, 858-864. 

M. Hmadeh, Z. Lu, Z. Liu, F. Gándara, H. Furukawa, S. Wan, V. Augustyn, R. Chang, L. Liao, F. Zhou, E. Perre, V. Ozolins, K. Suenaga, X. Duan, B. Dunn, Y. Yamamto, O. Terasaki and O. M. Yaghi, Chem. Mater., 2012, 24, 3511-3513.

21 W. H. Li, K. Ding, H. R. Tian, M. S. Yao, B. Nath, W. H. Deng, Y. Wang and G. Xu, Adv. Funct. Mater., 2017, 27, 1-7.

22 M. G. Campbell, S. F. Liu, T. M. Swager and M. Dinca, J. Am. Chem. Soc., 2015, 43, 1378013783.

M. K. Smith, K. E. Jensen, P. A. Pivak and K. A. Mirica, Chem. Mater., 2016, 28, 5264-5268.

M. S. Yao, X. J. Lv, Z. H. Fu, W. H. Li, W. H. Deng, G. D. Wu and G. Xu, Angew. Chemie - Int. Ed., $2017,56,16510-16514$.

V. Rubio-Giménez, M. Galbiati, J. Castells-Gil, N. Almora-Barrios, J. Navarro-Sánchez, G. Escorcia-Ariza, M. Mattera, T. Arnold, J. Rawle, S. Tatay, E. Coronado and C. Martí-Gastaldo, Adv. Mater., 2018, 30, 1-8.

27 B. Hoppe, K. D. J. Hindricks, D. P. Warwas, H. A. Schulze, A. Mohmeyer, T. J. Pinkvos, S. Zailskas, M. R. Krey, C. Belke, S. König, M. Fröba, R. J. Haug and P. Behrens, CrystEngComm, 2018, 20, 6458-6471.

M. Ko, A. Aykanat, M. K. Smith and K. A. Mirica, Sensors, 2017, 17, 2192.

X. Song, X. Wang, Y. Li, C. Zheng, B. Zhang, C. Di, F. Li, C. Jin, W. Mi, L. Chen and W. Hu, Angew. Chemie Int. Ed., 2020, 59, 1118-1123.

W. Zhou, S. Lv, X. Liu, Y. Li and J. Liu, Chem. Commun., 2019, 55, 11207-11210. 5058. Commun., 2019, 10, 1-10. 
34 A. Mahringer, A. C. Jakowetz, J. M. Rotter, B. J. Bohn, J. K. Stolarczyk, J. Feldmann, T. Bein and D. D. Medina, ACS Nano, 2019, 13, 6711-6719.

K. J. Erickson, F. Léonard, V. Stavila, M. E. Foster, C. D. Spataru, R. E. Jones, B. M. Foley, P. E. Hopkins, M. D. Allendorf and A. A. Talin, Adv. Mater., 2015, 27, 3453-3459.

X. Chen, Z. Wang, Z. M. Hassan, P. Lin, K. Zhang, H. Baumgart and E. Redel, ECS J. Solid State Sci. Technol., 2017, 6, P150-P153.

L. Sun, B. Liao, D. Sheberla, D. Kraemer, J. Zhou, E. A. Stach, D. Zakharov, V. Stavila, A. A. Talin, Y. Ge, M. D. Allendorf, G. Chen, F. Léonard and M. Dincă, Joule, 2017, 1, 168-177.

W. Ma, T. Miao, X. Zhang, K. Takahashi, T. Ikuta, B. Zhang and Z. Ge, Nanoscale, 2016, 8, 2704-2710. 63. $46,3185-3241$.

43 R. Ameloot, L. Stappers, J. Fransaer, L. Alaerts, B. F. Sels and D. E. De Vos, Chem. Mater., 2009, 21, 2580-2582.

U. Mueller, M. Schubert, F. Teich, H. Puetter, K. Schierle-Arndt and J. Pastré, J. Mater. Chem., 2006, 16, 626-636.

45 H. Al-Kutubi, J. Gascon, E. J. R. Sudhölter and L. Rassaei, ChemElectroChem, 2015, 2, 462-474.

46 T. W. and H. Z. J. He, H. Feng, T. Wang, Chin. J. Chem, 2018, 36, 31-36.

47 A. Martinez Joaristi, J. Juan-Alcañiz, P. Serra-Crespo, F. Kapteijn and J. Gascon, Cryst. Growth Des., 2012, 12, 3489-3498. 
51 V. Stavila, A. A. Talin and M. D. Allendorf, Chem. Soc. Rev., 2014, 43, 5994-6010.

52 S. Yadnum, J. Roche, E. Lebraud, P. Négrier, P. Garrigue, D. Bradshaw, C. Warakulwit, J. Limtrakul and A. Kuhn, Angew. Chemie, 2014, 126, 4082-4086.

53 O. Touayar, B. Rougié, J. M. Coutin and J. Bastie, Metrologia, 1998, 35, 387-391.

54 V. K. Thakur, D. Vennerberg, S. A. Madbouly and M. R. Kessler, RSC Adv., 2014, 4, 6677-6684.

55 M. S. Dresselhaus, G. Chen, M. Y. Tang, R. Yang, H. Lee, D. Wang, Z. Ren, J. P. Fleurial and P. Gogna, Adv. Mater., 2007, 19, 1043-1053.

56 S. H. Kang, S. H. Choi, M. S. Kang, J. Y. Kim, H. S. Kim, T. Hyeon and Y. E. Sung, Adv. Mater., $2008,20,54-58$.

57 G. J. Snyder and E. S. Toberer, Nat. Mater., 2010, 7, 101-110.

58 X. Lü and J. Chu, Phys. Status Solidi Basic Res., 2001, 225, 35-41.

59 K. Termentzidis, P. Chantrenne and P. Keblinski, Phys. Rev. B, 2009, 79, 1-9.

60 X. Zhang and X. Wu, Comput. Mater. Sci., 2016, 123, 40-43. 